\title{
The phenylhexyl isothiocyanate induces apoptosis and inhibits leukemia cell growth in vivo
}

\author{
LULU LU ${ }^{1,3}$, DELONG LIU ${ }^{1}$, XUdONG MA², ANASTASIA BEKLEMISHEV ${ }^{1}$, \\ KAREN SEITER $^{1}$, TAUSEEF AHMED ${ }^{1}$ and J.W. CHIAO ${ }^{1}$ \\ ${ }^{1}$ Department of Medicine, New York Medical College, Valhalla, NY, USA; ${ }^{2}$ Department of Hematology, Zhangzhou \\ Municipal Hospital, Zhangzhou; ${ }^{3}$ Department of Hematology, Henan Tumor Hospital, Zhengzhou, Henan, P.R. China
}

Received July 26, 2006; Accepted September 12, 2006

\begin{abstract}
Isothiocyanates are potent chemopreventive agents for carcinogen-induced cancers in rodents. The major mode of action for chemoprevention is cytoprotection i.e. inducing detoxifying enzymes to remove the carcinogens, thus blocking the initiation of carcinogenesis. Analysis has indicated that isothiocyanates also act at the post-initiation levels of carcinogenesis. We have also reported that the phenylhexyl isothiocyanate (PHI) induced growth arrest and apoptosis in human leukemia HL-60 cells in culture. Since then we have investigated the in vivo efficacy of PHI. The effects of PHI were evaluated in immunodeficient mice, with xenografts of human leukemia HL-60 cells. The maximum tolerated dose (MTD) was determined. The experimental mice received $80 \%$ of the MTD. Oral feedings of PHI significantly reduced tumor incidence $(\mathrm{p}<0.05)$ without overt toxicity. PHI inhibited cell cycle progression through the down-regulation of cyclin expression, $\mathrm{Rb}$ phosphorylation and the upregulation of the cdk-inhibitors. Apoptosis was significantly increased in the treated tumors but not in the normal mouse tissues. In conclusion, PHI induced apoptosis and inhibited the growth of xenografts by targeting the cell cycle regulators. PHI induced selective apoptosis effects in the rapidly growing tumor cells but not in the normal tissues.
\end{abstract}

\section{Introduction}

Natural isothiocyanates occur as glucosinolates in cruciferous vegetables such as broccoli, cabbage, watercress, and kales (1). Isothiocyanates are released upon enzyme hydrolysis when the vegetables are cut or masticated (2). The natural or synthetic isothiocyanate compounds are potent cancer

Correspondence to: Dr Delong Liu or Professor J.W. Chiao, Department of Medicine, New York Medical College, Valhalla, NY 10595, USA

E-mail: Delong_Liu@nymc.edu Jen-Wei_Chiao@nymc.edu

Key words: phenylhexyl isothiocyanate, selective inhibition, leukemia, mice chemopreventive agents, as demonstrated in a number of carcinogen-induced rodent cancer models (3-5). The main chemopreventive mechanism is cytoprotection, i.e. inhibiting the cytochrome P450S and inducing detoxifying enzymes, thus blocking the initiation of carcinogenesis $(6,7)$.

Certain analyses have indicated that isothiocyanates may also act at the post-initiation levels. Our laboratory has also reported that the phenylhexyl isothiocyanate and sulforaphane induced cell cycle arrest and apoptosis in prostate cancer cells in culture, as well as in xenografted tumors (8-11). Conaway et al (12) described that the malignant progression of mouse lung adenocarcinoma could be blocked by dietary isothiocyanates. Isothiocyanates have also been reported to inhibit the proliferation of human leukemia cell lines. A number of isothiocyanates, including sulforaphane and 2oxohexyl (13), benzyl (14) and the allyl isothiocyanates (15) have been shown to mediate cell cycle arrest and apoptosis in myeloid and $\mathrm{T}$ cell leukemia cells in culture. However, the in vivo effects of isothiocyanates on the growth of leukemia cells remain unclear.

In this study, we examined the effects of the phenylhexyl isothiocyanate (PHI), a synthetic compound with a sixcarbon arylalkyl chain, on the growth of xenografted tumors of human leukemia HL-60 cells in immunodeficient mice. Among the known isothiocyanates, PHI is considered to be among the most potent for inhibiting carcinogen-initiated lung tumors in mice (16). We have recently demonstrated that PHI induces growth arrest and apoptosis in leukemia HL-60 cells in culture, involving a mechanism that inhibits the activity of histone deacetylases (17). Dietary PHI was further observed to reduce the incidence of spontaneous leukemia in Fischer rats (18). However, it was not determined whether the mechanism of the inhibition of spontaneous leukemia was due to a chemotherapeutic effect of PHI. In the present study, we demonstrated that PHI significantly inhibited the xenografts of human leukemia cells by inducing cell cycle arrest and apoptosis. It was further demonstrated that PHI may have a selective activity in inducing apoptosis in the tumor cells, but not in the normal mouse tissues.

\section{Materials and methods}

Chemicals and cell culture. PHI, with $>98 \%$ purity, was purchased from the LKT Laboratory (St. Paul, MN, USA). The 
human leukemia HL-60 cell line was maintained in RPMI1640 medium supplemented with $15 \%$ heat-inactivated fetal calf serum and $1 \%$ penicillin/streptomycin. The cultures were maintained at $37^{\circ} \mathrm{C}$ with $5 \% \mathrm{CO}_{2}$.

Maximum tolerated dosage assay (MTD). To optimize the dosage of PHI, the MTD was determined. Groups of 7 male 5-week-old BALB/c nu/nu mice (Charles River Laboratories, Wilmington, MA, USA) were provided with $10 \mu \mathrm{mol}, 15 \mu \mathrm{mol}$ or $20 \mu \mathrm{mol}$ PHI in corn oil by gavage $(0.15 \mathrm{ml} / \mathrm{mouse})$, daily for a total of 4 weeks. The control group was provided with corn oil in the same quantity without PHI. The body weights of the mice were determined twice/week. The MTD was defined as the highest dosage that caused not $>10 \%$ mean weight loss when compared with the mean body weight of the control group. Eighty percent of the MTD was used as the PHI dosage.

Xenograft tumor assays. Five-week-old BALB/c (nu/nu) male mice were housed in a barrier facility with 12-h light/dark cycles; tap water and mouse diets were provided ad libitum. The xenografted tumors were established by a single subcutaneous injection at the flank with $1 \times 10^{6} \mathrm{HL}-60$ cells in $0.15 \mathrm{ml}$ RPMI-1640 medium. The xenografted animals were randomly divided into two groups with 17 mice in each group. Each mouse from the experimental group was provided by gavage $(0.15 \mathrm{ml})$ with $12 \mu \mathrm{mol} \mathrm{PHI}$ in corn oil per day. The control group of mice received the same quantity of corn oil as a vehicle control. The PHI was prepared twice/week and kept under nitrogen gas at $-80^{\circ} \mathrm{C}$. The mice were fed with PHI or the control vehicle, starting 2 days after the inoculation of the HL-60 cells, and this continued until the termination of the experiments. The animal body weights were recorded weekly and at necropsy. At necropsy, the weight of the xenografted tumors and spleens was determined. Parts of the tumors, spleens and livers were frozen in liquid nitrogen, and a piece was fixed in $10 \%$ formalin and embedded in paraffin.

Apoptosis and protein expression. The apoptotic cells of the xenografted tumors, livers and spleens were determined on paraffin-embedded tissue slides by the presence of DNA strand breaks, using terminal deoxynucleotidyl transferasemediated biotinylated UTP nick end-labeling (TUNEL). An in situ detection kit from Roche Molecular Biochemicals (Mannheim, Germany) was employed according to the manufacturer's directions (8). Briefly, the tumor sections were rehydrated and incubated with protease K. After incubating with the TUNEL reaction and staining with the appropriate substrate, the presence of apoptotic cells was determined under a light microscope. To enumerate the apoptotic cells, six different fields on each section were examined. At least 100 cells from each field were counted. The mean populations of apoptotic cells per section from the control group and experimental group were reported.

The protein levels of the xenografts were determined by Western blot analyses using standard procedures as described previously (8). Total proteins of equal weight were prepared from each group of pooled individual xenografts. The xenografts were homogenized in the presence of a lysis buffer

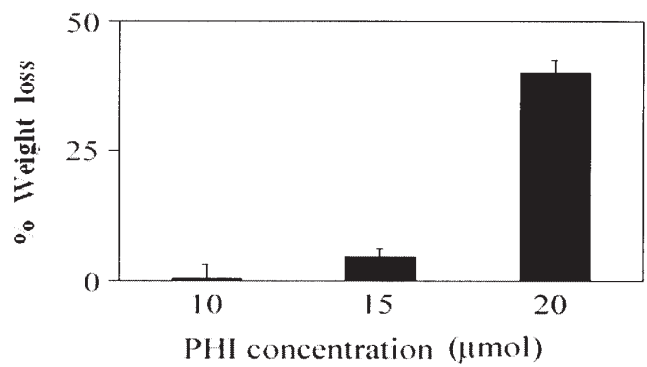

Figure 1. The maximum tolerated dosage (MTD) of PHI in immunodeficient mice. Seven mice in each group received corn oil without PHI (vehicle control) or PHI at 10,15 or $20 \mu \mathrm{mol} /$ mouse by gavage according to the procedure described in Materials and methods. Body weight was determined 4 weeks after the initiation of the experiment. We show the percentage of mean body weight loss of the mice that received PHI, as compared to the mean body weight of the mice that received corn oil without PHI. The vertical bars indicate the mean values $\pm \mathrm{SD}$.

with freshly prepared protease inhibitors, and the lysates were collected after centrifugation at $4^{\circ} \mathrm{C}$. Equal amounts of lysate proteins were separated by $10 \%$ SDS-PAGE, electrotransferred to a nitrocellulose membrane, and immunoblotted. Antibodies against the cell cycle and apoptosis regulators, including the proliferating cell nuclear antigen (PCNA), p21, p27, cyclin D1 and Rb, as well as bcl-2, caspase-9 and poly ADP-ribose polymerase (PARP) were purchased from Santa Cruz Biotechnology (Santa Cruz, CA, USA). The appropriate HRP conjugated-secondary antibodies were used and the reactive proteins were visualized using an enhanced chemiluminescence kit (Amersham Pharmacia Biotech, Piscataway, NJ, USA). Each membrane was stripped and reprobed with an anti- $\beta$-actin antibody as a loading control. The measurements of protein expression were based on densitometry determinations.

Statistics. The mean values of body, tumor and spleen weight, and apoptotic cells from the control or experimental groups were compared with the Student's t-test, with a pvalue of $\mathrm{p}<0.05$ considered as statistically significant.

\section{Results}

PHI inhibited growth of xenografts. The MTD assay was performed to determine the optimal PHI dosage to be used for the experimental group of immunodeficient mice. The dosage of $12 \mu \mathrm{mol} / \mathrm{mouse}, 80 \%$ of the MTD $(15 \mu \mathrm{mol} /$ mouse), was selected (Fig. 1).

The effects of PHI on the growth of xenografted tumors of HL-60 cells in immunodeficient mice were evaluated. The animals were observed throughout the study and they showed no signs of unusual behavior with the PHI feeding. Sixteen out of the 17 mice from the control group developed palpable tumors 14 days after the transplantation of the HL-60 cells (94.1\% tumor incidence). The PHI-treated mice had a tumor incidence of $58.5 \%$, significantly less than the control group $(\mathrm{p}=0.005)$. The tumor volumes of the PHI-treated group were initially smaller, as compared with the control tumors, and this difference persisted during the study period. Fig. 2A shows that PHI suppressed tumor growth. The mean tumor weight, determined at necropsy, in the control mice was 
A

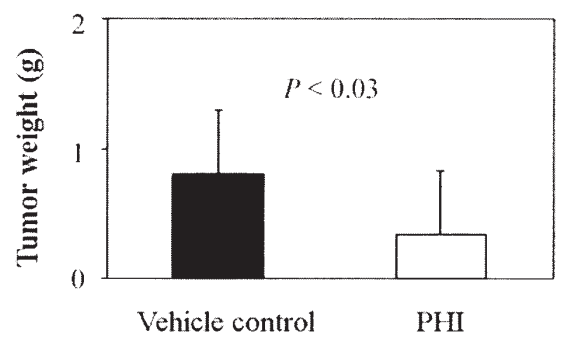

B

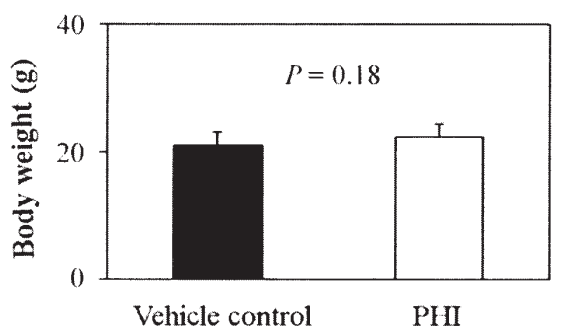

C

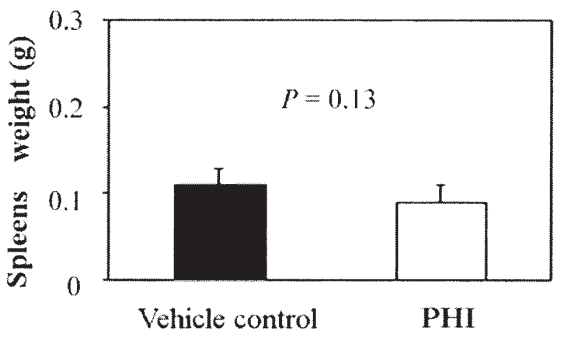

Figure 2. PHI administration inhibited the growth of xenografted tumors of HL-60 cells in immunodeficient mice. (A) The mean xenograft weight from the mice that received PHI ( $\square$ ), or the vehicle control (ם) at necropsy. (B) The mean body weight of the mice that received PHI $(\square)$, or the vehicle control (ם) at necropsy. (C) The mean spleen weight from the mice that received PHI $(\square)$, or the vehicle control ( $\square$ ) at necropsy. The vertical bars are the means \pm SD. The student's t-test was used for a comparison of the two groups.

$0.8 \pm 0.5 \mathrm{~g}$, as compared to $0.35 \pm 0.48 \mathrm{~g}$ in the PHI-treated mice. This reflects a $44.4 \%$ reduction in tumor weight in the PHItreated mice $(p<0.03)$. The body weight of the mice was recorded to determine if the inhibition of the tumor xenograft growth was due to weight loss. As depicted in Fig. 2B, the mean body weight of the control $(21.29 \pm 1.67 \mathrm{~g})$ and PHItreated mice $(22.4 \pm 2.05 \mathrm{~g})$ did not differ significantly at necropsy $(p=0.18)$, which could not account for the tumor inhibition. Fig. 2C shows that the mean weight of the spleens from the PHI-treated mice $(0.09 \pm 0.02 \mathrm{~g})$ was comparable to that of the controls $(0.11 \pm 0.02 \mathrm{~g})$. The difference in weight was not significant $(\mathrm{p}=0.13)$, suggesting that there was no overt toxicity with the PHI feeding.

PHI inhibited cell cycle progression. Since the PHI-treated xenografted tumors became smaller than the controls, the proliferation of the tumor cells was evaluated. The levels of the cell cycle regulators from the xenografted tumors were examined by Western blot analysis. Fig. 3 shows that the proliferation marker PCNA was significantly reduced in the pooled PHI-treated tumors when compared with the controls.

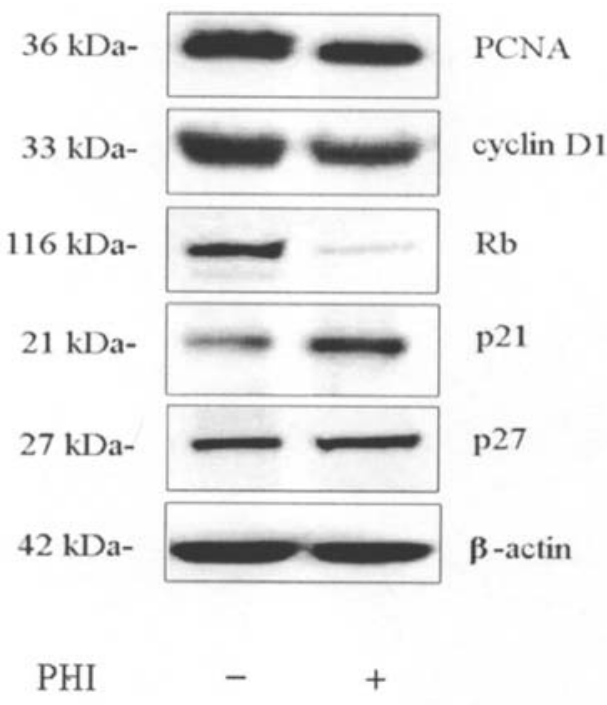

Figure 3. PHI inhibited cell cycle progression. Western blots were performed with pooled total proteins from the tumor tissues of each experimental group, obtained at necropsy, as described in Materials and methods. Representative blots from three separate experiments are shown. Antibodies against PCNA, cyclin D1 (cross-reactive with D2 and D3), Rb related proteins, $\mathrm{p} 21, \mathrm{p} 27$ or $\beta$-actin (the loading control) were used.

Since the induction of cyclin-dependent kinase (cdk) inihibitors, or the decrease of cyclins can both lead to a decreased cdk activity, the levels of the kinase inhibitors p21 and p27, as well as cyclin D1 were assessed. Fig. 3 shows that increased levels of p21 and p27 (12\% and 38\%), along with a reduced expression of cyclin D1 $(21 \%)$, were determined in the PHI-treated tumors when compared with the controls (Fig. 3). The PHI effects were then examined on the phosphorylation of $\mathrm{Rb}$. This is because an induction of cdk inhibitors or a decrease in cyclins can lead to a decreased cdk activity, which affects the down-stream phosphorylation of $\mathrm{Rb}$ proteins. Fig. 3 shows a significant reduction of $\mathrm{Rb}$ phosphorylation in the PHI-treated tumors as compared with the controls.

Apoptosis induction. Apoptotic cells, with the morphology of a condensed cytoplasm and pyknotic hyperchromatic nuclei, were evident in the sections of the xenografts. To quantify the apoptotic cells, those cells with DNA strand breaks were determined in situ using the TUNEL assay. Fig. 4A shows a typical image of the apoptotic cells with TUNEL staining in the PHI-treated tumor. In contrast, an image of a control tumor shows significantly fewer apoptotic cells (Fig. 4B). Fig. $4 \mathrm{C}$ shows that the average number of apoptotic cells in the PHI-treated tumors was $\sim 14.75 \%$, as compared with $1.33 \%$ in the control tumors, representing an $\sim 10$-fold increase $(\mathrm{p}=0.003)$. Apoptosis was further analyzed by the cleavage of PARP, a target of proteolysis of caspases that execute apoptosis, with Western blot analyses. A significant decrease, $43 \%$, of the full length PARP was detected in the PHI-treated tumors as compared with the untreated control group (Fig. 4D). The effectors of apoptosis, caspases and the apoptotic repressor bcl-2 proteins, were further examined by Western blot analysis. A significant reduction in the bcl-2 level $(33 \%)$ along with an increased expression of caspase-9 

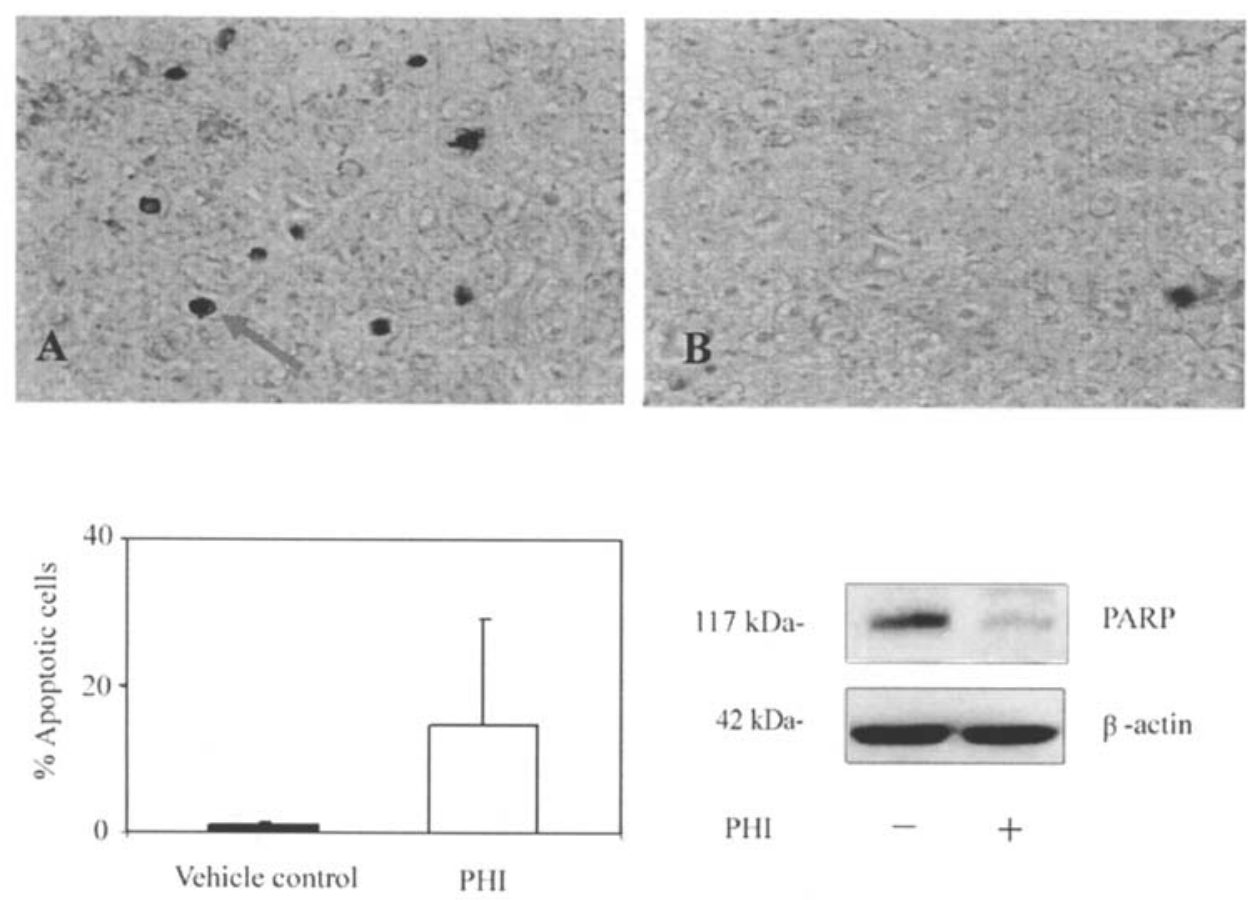

PARP

C

D

Figure 4. Apoptosis in the xenografted tumors from immunodeficient mice at necropsy. (A) In situ detection of DNA fragmentation by TdT-mediated dUTP nick end-labeling (TUNEL) in a PHI-treated tumor. The arrow indicates the positively stained apoptopic cells. (B) TUNEL assay of a tumor from the control group. (C) The mean of the apoptotic cells in the tumors from the PHI-treated mice ( $\square$ ) as compared with those from the mice in the control group (ロ). The vertical bars represent the means \pm S.D. of apoptotic cells in the tumors, and the student's t-test was used for a comparison of the two groups. (D) The PARP protein was degraded in the tumor lysates from the PHI group. The illustrations shown in A, B and D were from the same PHI-treated and control mice.

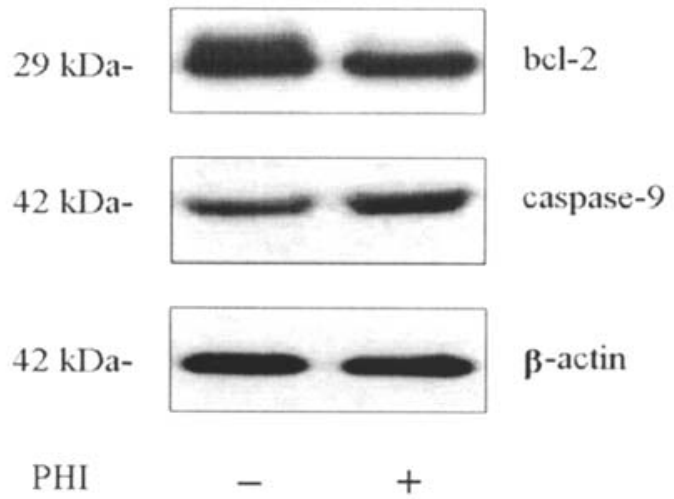

Figure 5. The alteration of the expression of bcl-2 and caspase-9. Pooled total proteins from the tumor tissues of each experimental group were obtained at necropsy as described in Materials and methods. Representative blots from three separate experiments are shown. Antibodies against bcl-2, caspase-9 or $\beta$-actin (the loading control) were used.

$(31 \%)$, were detected in the pooled PHI-treated tumors when compared with the control group (Fig. 5).

To evaluate the potential effects of PHI on normal tissues, the mouse organs and the connective tissue surrounding the xenografts were examined with TUNEL assay, to evaluate whether PHI induced apoptosis. Few apoptotic cells were seen in the livers and their quantities were similar between the control mice $(0.018 \pm 0.001 \%)$ and the PHI-treated mice $(0.02 \pm 0.001 \%)(\mathrm{p}>0.05)$. Similarly, there was no difference in the number of apoptotic cells in the spleens from the PHI- treated and control mice. The connective tissues surrounding the tumors were also examined for apoptosis. In contrast to the tumors, there was no increase in the apoptotic cells detected in these normal tissues.

\section{Discussion}

This study demonstrated that PHI effectively inhibited the growth of xenografted tumors of human leukemia HL-60 cells in immunodeficient mice, with no evidence of detectable toxicity to normal tissues. The inhibitory effects of PHI were demonstrated at both the molecular and tumor levels. PHI induced a significant reduction in tumor incidence, as well as in the volume and weight of the xenografts. The inhibition of the rapidly growing tumor cells indicated that PHI acts at the post-initiation levels of the progression stages of leukemia cells.

It is known that the key components in cell cycle regulation are the cyclins, the cyclin-dependent kinases (cdks), and their inhibitors (19). Specific cyclin/cdk complexes formed at specific stages of the cell cycle and their activities are required for the progression of the cycle through the Sphase and mitosis. Several cdk inhibitors have been demonstrated to mediate cell cycle arrest following growth inhibitory stimuli. Members of the kinase inhibitor protein family, such as p21 and p27, bind and inhibit target cyclin/ cdk activities, thus leading to growth arrest (20). In this study, the data showed clearly that PHI down-regulated cyclin D1, and induced the expression of the cdk inhibitors p21 and p27. The elevated levels of the cdk inhibitors could 
increase their binding with the effector cyclin/cdk complexes, thus inhibiting the kinase activity. As a result, the $\mathrm{Rb}$ proteins, which activate cell cycle progression, diminished. Taken together, as the PCNA expression decreased, the data indicated that PHI induced cell cycle arrest, probably including a blocking of the G1- to S-phase transition. Similar events of cell cycle arrest, mediated by isothiocyanates, have also been reported in experiments performed in culture and in xenografts $(8,9)$.

The inhibition of xenografts was also attributed to apoptosis, induced by PHI. The increase of apoptotic cells with DNA strand breaks in the tumors, the cleavage of PARP, and the down-regulation of bcl-2 indicated that apoptotic cell death was a critical event in tumor inhibition. Previous studies have described that some isothiocyanates are capable of inducing apoptosis in HL-60 in culture, with the activation of caspases-3, -8 and $-9(14,21)$. However, there is not much information regarding the apoptosis pathway induced in vivo by the isothiocyanates. Since our study revealed an involvement of caspase- 9 and bcl-2 expression, the results suggest a mediation of the mitochondrial pathway of apoptosis. Since the primary cause of treatment failures in acute leukemia is frequently associated with a defect in the mitochondrial-mediated apoptotic pathway involving the bcl-2 family (22), our results indicate that PHI could be a potential agent in activating apoptosis in leukemia cells.

In this study, the mice showed no signs of unusual behavior with PHI feeding. There was no detectable evidence of overt toxicity as evaluated by body and organ weight. Apoptosis analysis further revealed that PHI did not induce apoptosis in the normal mouse organs and tissues. We have previously demonstrated that PHI mediated apoptosis in HL-60 cells in culture, but not in the mononuclear cells from normal peripheral blood and bone marrow (17). Therefore, the experimental results strongly suggest that PHI may preferentially induce apoptosis in leukemia cells in vivo, with minimal toxicity to normal cells. The basis of this selectivity against leukemia cells has not been fully elucidated. One reason could be that the xenografted tumors are composed of rapidly proliferating leukemia cells, which may be more sensitive to PHI's effects. This is supported by the fact that PHI targets cell cycle regulators for proliferation cessation. This observation is significant since there are not many agents possessing the selective activity to eliminate only leukemia cells but not to be toxic to normal cells. Further analyses are needed to elucidate the cellular and molecular basis regarding the preferential activity of PHI against leukemia cells.

\section{Acknowledgements}

This research is supported by a research grant from the US Army, the Blood Diseases Research Fund of the New York Medical College, and by a generous grant from the Cantigny Research Foundation Inc., Marietta, GA, USA. We thank Ms. Ruth Gallagher for proofreading the manuscript.

\section{References}

1. Josephsson E: Distribution of thioglucosides in different parts of Brassica plants. Phytochemistry 5: 1617-1627, 1967.
2. Fenwick GR, Heaney RK and Mullin WJ: Glucosinolates and their breakdown products in food and food plants. Crit Rev Food Sci Nutr 18: 123-201, 1983.

3. Hecht SS: Chemoprevention by isothiocyanates. J Cell Biochem Suppl 22: 195-209, 1995.

4. Jiao D, Ho CT, Foiles P and Chung FL: Identification and quantification of the $\mathrm{N}$-acetylcysteine conjugate of allyl isothiocyanate in human urine after ingestion of mustard. Cancer Epidemiol Biomarkers Prev 3: 487-492, 1994.

5. Wattenberg LW: Perspectives in cancer research: chemoprevention of cancer. Cancer Res 45: 1-8, 1985.

6. Zhang Y and Talalay P: Anticarcinogenic activities of organic isothiocyanates: chemistry and mechanisms. Cancer Res 54: S1976-S1981, 1994

7. Yang CS, Smith TJ and Hong JY: Cytochrome P-450 enzymes as targets for chemoprevention against chemical carcinogenesis and toxicity: opportunities and limitations. Cancer Res 54: S1982-S1986, 1994.

8. Chiao JW, Hongyan W, Gita R, Conaway CC, Funglung C, Longgui $\mathrm{W}$ and Delong L: Ingestion of an isothiocyanate metabolite from cruciferous vegetables inhibits growth of human prostate cancer cell xenografts by apoptosis and cell cycle arrest. Carcinogenesis 25: 1403-1408, 2004.

9. Chiao JW, Chung FL, Kancherla R, Ahemad T, Mettelman A and Conaway CC: Sulforaphane and its metabolite mediate growth arrest and apoptosis in human prostate cancer cells. Int J Oncol 20: 631-636, 2002.

10. Wang L, Delong L, Ahmed T, Chung FL, Conaway CC and Chiao JW: Targeting cell cycle machinery as a molecular mechanism of sulforaphane in prostate cancer prevention. Int J Oncol 24: 187-192, 2004.

11. Chiao JW, Chung FL, Krzeminski J, Amin S, Ashad R, Ahmed T and Conaway CC: Modulation of growth of prostate cancer cells by the $\mathrm{N}$-acetylcysteine conjugate of phenethyl isothiocyanate. Int J Oncol 16: 1215-1219, 2000

12. Conaway CC, Wang CX, Pittman B, Yang YM, Schwartz JE, Tian D, McIntee EJ, Hecht SS and Chung FL: Phenethyl isothiocyanate and sulforaphane and their $\mathrm{N}$-acetylcysteine conjugates inhibit malignant progression of lung adenomas induced by tobacco carcinogens in A/J mice. Cancer Res 65 : 8548-8557, 2005.

13. Irena M, Katarzyna S and Teresa KG: Sulforaphane and 2oxohexyl isothiocyanate induce cell growth arrest and apoptosis in L-1210 leukemia and ME-18 melanoma cells. Oncol Rep 10: 2045-2050, 2003

14. Noriyuki M, Koji U, Toshihiko O and Yoshimasa N: A link between benzyle isothiocyanate-induced cell cycle arrest and apoptosis: involvement of mitogen-activated protein kinases in the bcl-2 phosphorylation. Cancer Res 64: 2134-2142, 2004.

15. Zhang YS, Li T and Veronica G: Selected isothiocyates rapidly induce growth inhibition of cancer cells. Mol Cancer Ther 2: 1045-1052, 2003

16. Jiao D, Smith TJ, Yang CS, Pittman B, Desai D, Amin S and Chung FL: Chemopreventive activity of thiol conjugates of isothiocyanates for lung tumorigenesis. Carcinogenesis 18: 2143-2147, 1997.

17. Ma XD, Fang YQ, Anastasia B, Wei D, Feng JY, Ahmed T, Delong L and Chiao JW: Phenylhexyl isothiocyanate inhibits histone deacetylases and remodels chromatins to induce growth arrest in human leukemia cells. Int J Oncol 28: 1287-1293, 2006.

18. Chung FL, Kelloff G, Steele V, Pittman B, Zang E, Jiao D, Rigotty J, Choi CI and Rivenson A: Chemopreventive efficiency of Arylalky isothiocyanates and N-acetylcysteine for lung tumorigenesis in Fischer rats. Cancer Res 56: 772-778, 1996.

19. Sherr CJ and Roberts JM: Inhibitors of mammalian G1 cyclindependent kinases. Genes Dev 9: 1149-1163, 1995.

20. Sherr CJ and Roberts JM: CDK inhibitors: positive and negative trgulators of G1-phase progression. Genes Dev 13; 1501-1502, 1999.

21. Xu K, Paul J and Thornalley: Involvement of glutathione metabolism in the cytotoxicity of the phenethyl isothiocyanate and its cysteine conjugate to human leukaemia cells in vitro. Biochem Pharmacol 61: 165-177, 2001.

22. Del Principe MI, Del Poeta G, Venditti A, Buccisano F, Maurillo L, Mazzone C, Bruno A, Neri B, Irno Consalvo M, Lo Coco F and Amadori S: Apoptosis and immaturity in acute myeloid leukemia. Hematology 10: 25-34, 2005. 